\title{
Stability of MOF-5 in a Hydrogen Gas Environment Containing Fueling Station Impurities
}

Yang Ming ${ }^{\mathrm{a},}$ Justin Purewal ${ }^{\mathrm{b}}$, Jun Yang ${ }^{\mathrm{b}}$, Chunchuan $\mathrm{Xu}^{\mathrm{b}}$, Andy Drews ${ }^{\mathrm{b}}$, Mike Veenstra $^{\mathrm{b}}$, Manuela Gaab ${ }^{\mathrm{c}}$, Ulrich Müller ${ }^{\mathrm{c}}$, and Donald J. Siegel ${ }^{\mathrm{d}, *}$

${ }^{a}$ Department of Physics, University of Michigan, 1440 Randall Laboratory, 450 Church St., Ann Arbor, MI 48109-1040, USA

${ }^{b}$ Ford Motor Company, Research and Advanced Engineering, Dearborn, MI 48121, USA

${ }^{c}$ BASF SE, Process Research and Chemical Engineering, 67056 Ludwigshafen, Germany

${ }^{\mathrm{d}}$ Mechanical Engineering Department, University of Michigan, 2250 G.G. Brown Laboratory, 2350 Hayward St., Ann Arbor, MI 48109-2125, USA

Abstract: Metal-organic frameworks (MOFs) are an emerging class of porous, crystalline materials with potential application as hydrogen storage media in fuel cell vehicles. Unlike lower capacity adsorbents such as zeolites and carbons, some MOFs are expected to degrade due to attack by impurities present in the hydrogen fuel stream. Hydrogen intended for use in fuel cell vehicles should satisfy purity standards, such as those outlined in SAE J2719. This standard limits the concentration of certain species in the fuel stream based primarily on their deleterious effects on PEM fuel cells. However, the impact of these contaminants on MOFs is mostly unknown. In the present study MOF-5 is adopted as a prototypical moisture-sensitive hydrogen storage material. Five "impure" gas mixtures were prepared by introducing low-to-moderate levels (i.e., up to 200 times greater than the $\mathrm{J} 2719$ limit) of selected contaminants $\left(\mathrm{NH}_{3}, \mathrm{H}_{2} \mathrm{~S}\right.$, $\mathrm{HCl}, \mathrm{H}_{2} \mathrm{O}, \mathrm{CO}, \mathrm{CO}_{2}, \mathrm{CH}_{4}, \mathrm{O}_{2}, \mathrm{~N}_{2}$, and $\mathrm{He}$ ) to pure hydrogen gas. Subsequently, MOF-5 was exposed to these mixtures over hundreds of adsorption/desorption pressure-swing cycles and for extended periods of static exposure. The impact of exposure was assessed by periodically measuring the hydrogen storage capacity of an exposed sample. Hydrogen chloride was observed to be the only impurity that yielded a measurable, albeit small, decrease in hydrogen capacity; no change in $\mathrm{H}_{2}$ uptake was observed for the other impurities. Post-cycling and post-storage MOF-5 samples were also analyzed using infrared spectroscopy and x-ray diffraction. These analyses reveal slight changes in the spectra for those samples exposed to $\mathrm{HCl}$ and $\mathrm{NH}_{3}$ compared to the pristine material. These measurements suggest that MOF-5 - and likely many other MOFs exhibit sufficient robustness to withstand prolonged exposure to 'off-spec' hydrogen fuel.

KEYWORDS: Hydrogen storage, Metal-organic frameworks, robustness, impurities, fuel cell poisoning.

\section{Introduction}

Physical adsorption (alternatively referred to as physisorption or cryo-adsorption) is a hydrogen storage method involving the adsorption of $\mathrm{H}_{2}$ in the micropores of highly porous materials such as active carbons and zeolites. At low temperatures the hydrogen density within 
micropores can significantly exceed that of the bulk gas. Since the adsorbed hydrogen does not dissociate or form new chemical bonds, this storage approach is characterized by a low enthalpy change, which reduces heat management issues, and rapid kinetics. Another perceived advantage of cryo-adsorbents over other hydrogen storage materials (e.g., metal hydrides, complex hydrides) is their chemical stability with respect to gas impurities and moisture.

Metal-organic frameworks (MOFs), a relatively new class of synthetic, microporous materials, are leading candidates for physisorption-based hydrogen storage. Their tunable pore structure creates the potential for achieving micropore volumes $(>1.5 \mathrm{~L} / \mathrm{kg})$ that are significantly larger than those in conventional adsorbents [1-3]. However, while most conventional adsorbents such as carbons and zeolites are chemically stable (requiring only regeneration to remove adsorbed water and other impurities), MOFs may be less robust against common impurities in hydrogen fuel streams. For example, zinc-based MOFs with carboxylate linkers (e.g., MOF-5) are known to be susceptible to chemical degradation in humid conditions, owing to the weak metal-ligand bond between $\mathrm{Zn}$ and O [4-11]. Studies have also identified MOFs which are unstable in the presence of high concentrations of hydrogen sulfide[12-14], ammonia[15-17], and other common impurities in industrial gas streams [18, 19]. In most cases, MOF degradation is manifested as a decrease in storage capacity due to a reduction in the surface area and pore volume, a change in long-range crystal structure, and/or alteration in local chemical bonding $[7,10,11,20]$

While most studies have focused on scenarios in which MOFs are exposed to high concentrations of harmful impurities, these environments are far more severe than what would be realistically encountered in an on-board hydrogen storage system. As is the case for semiconductor and $\mathrm{Li}$-ion battery production, environmental controls can be implemented during production and packaging. Once installed in a vehicle, the most likely scenarios for the exposure of hydrogen storage materials to impurities would be via the hydrogen fuel stream itself, or from exposure to the atmosphere due to leakage during refueling.

Hydrogen gas from fueling stations is required in the U.S. to be compliant with fuel quality standards outlined in the SAE J2719 specification [21]. (The international standard, ISO 14687-2, stipulates an identical purity benchmark.) Impurity limits set by J2719 are driven primarily by the need to protect the catalyst and polymer electrolyte components in the fuel cell stack. As a result, threshold limits for ammonia $(0.1 \mathrm{ppm})$, carbon monoxide $(0.2 \mathrm{ppm})$, sulfur species $(0.004 \mathrm{ppm})$, and halogenates $(0.05 \mathrm{ppm})$ are exceptionally stringent. Complete threshold limits are summarized in Table 1. Although SAE J2719 does recognize some impurities that are known to poison and/or deactivate conventional metal hydride-based storage materials (e.g., $\mathrm{CO}$ and $\mathrm{O}_{2}$ ), the impact of impurities on adsorbent materials such as MOFs are currently not accounted for. 
Table 1. Impurity test gas mixtures used in this study.

\begin{tabular}{|c|c|c|c|c|c|c|}
\hline $\begin{array}{c}\text { Gas } \\
\text { Mixture } \\
\text { No. }\end{array}$ & Impurity & $\begin{array}{c}\text { Test Gas } \\
\text { Concentration } \\
(p p m)\end{array}$ & $\begin{array}{l}\mathrm{J} 2719 \\
\text { Limit } \\
(p p m)\end{array}$ & $\begin{array}{l}\text { Cycle Test } \\
\text { Performed }\end{array}$ & $\begin{array}{c}\text { Storage } \\
\text { Test } \\
\text { Performed }\end{array}$ & $\begin{array}{c}\text { Impurity } \\
\text { concentration } \\
\text { relative to } \\
\text { J2719 } \\
\end{array}$ \\
\hline 1 & $\mathrm{NH}_{3}$ & 7 & 0.1 & Y & $\mathrm{N}$ & 70 \\
\hline 2 & $\mathrm{H}_{2} \mathrm{~S}$ & 1 & $0.004^{\mathrm{a}}$ & Y & Y & 250 \\
\hline 3 & $\mathrm{HCl}$ & 9 & $0.05^{\mathrm{b}}$ & $Y$ & $\mathrm{~N}$ & 180 \\
\hline 4 & $\mathrm{H}_{2} \mathrm{O}$ & 8 & 5 & Y & Y & 1.6 \\
\hline \multirow{6}{*}{5} & $\mathrm{CO}$ & 2 & 0.2 & \multirow{6}{*}{$\mathrm{Y}$} & \multirow{6}{*}{$\mathrm{N}$} & 10 \\
\hline & $\mathrm{CO}_{2}$ & 5.7 & 2 & & & 2.85 \\
\hline & $\mathrm{CH}_{4}$ & 8.3 & $2^{\mathrm{c}}$ & & & 4.15 \\
\hline & $\mathrm{O}_{2}$ & 9.6 & 5 & & & 1.92 \\
\hline & $\mathrm{N}_{2}$ & 119 & 100 & & & 1.19 \\
\hline & $\mathrm{He}$ & 505 & 300 & & & 1.7 \\
\hline $\begin{array}{c}\text { Not } \\
\text { Tested }\end{array}$ & $\mathrm{HCHO}$ & - & 0.01 & - & - & \\
\hline $\begin{array}{c}\text { Not } \\
\text { Tested }\end{array}$ & $\mathrm{HCOOH}$ & - & 0.2 & - & - & \\
\hline \multicolumn{7}{|c|}{$\begin{array}{l}{ }^{\text {a }} \text { Total sulfur category in J2719 includes hydrogen sulfide }\left(\mathrm{H}_{2} \mathrm{~S}\right) \text {, carbonyl sulfide }(\mathrm{COS}) \text {, carbon } \\
\text { disulfide }\left(\mathrm{CS}_{2}\right) \text { and mercaptans }\end{array}$} \\
\hline \multicolumn{7}{|c|}{$\begin{array}{l}{ }^{b} \text { Total halogenates category in J2719 includes hydrogen bromide }(\mathrm{HBr}) \text {, hydrogen chloride }(\mathrm{HCl}) \text {, } \\
\text { chlorine }\left(\mathrm{Cl}_{2}\right) \text { and organic halides (R-X) }\end{array}$} \\
\hline
\end{tabular}

The identity and concentration of impurities in the hydrogen fuel stream depends on the hydrogen production method. For example, hydrocarbons, carbon monoxide, and sulfur species are more likely to be present in hydrogen produced by steam methane reformation, the predominant method for hydrogen production at present. Field data from existing hydrogen stations confirms that the J2719 impurity limits are achievable, although some station samples still exceed the requirements [22]. Nitrogen, oxygen, and water are contaminants likely to leak in from the atmosphere during refueling process itself.

To our knowledge, no systematic studies exist regarding the chemical stability of MOFs after exposure to hydrogen streams containing impurities identified in J2719. The use pattern of the hydrogen storage system will strongly influence the mechanism by which degradation can occur. For example, if a MOF-based system is cycled frequently at cryogenic temperatures (i.e. cycling), it may accumulate the more strongly-bound impurity species by selective adsorption, 
potentially reducing the available storage capacity for hydrogen molecules. However, chemical degradation side-reactions involving the adsorbed impurities will proceed slowly at cryogenic temperatures. For MOFs stored at higher temperatures for extended periods (i.e. storage), chemical side-reactions will proceed faster, but the accumulation of impurities will be considerably smaller.

For this study we have chosen MOF-5 as a representative moisture-sensitive MOF for impurity testing. Alternatively designated as IRMOF-1, MOF-5 has the chemical formula $\mathrm{Zn}_{4} \mathrm{O}(\mathrm{bdc})_{3}$, where 'bdc' represents the 1,4 benzenedicarboxylate linker derived from industrially produced terephthalic acid. Extensive published work exists on both its hydrogen uptake capacity [10, 23-26] and thermal properties [27-29]. MOF-5 remains an attractive hydrogen storage material due to its balanced gravimetric and volumetric hydrogen uptake [30]. As a zinc-based MOF with a carboxylate linker, MOF-5 is susceptible to moisture-induced degradation. Of particular importance for this study is the fact that MOF-5 synthesis methods have been optimized, meaning that high-quality, fully-desolvated samples of MOF-5 are commercially available[1]. This is important because it enables the systematic study of impurity-induced degradation, without having to account for degradation caused or accelerated by poor sample quality.

In this study we examine the degradation potential of 10 impurities listed in SAE J2719. These impurities were mixed with nominally pure hydrogen gas at a concentration of up to several ppm, and include: $\mathrm{NH}_{3}, \mathrm{H}_{2} \mathrm{~S}, \mathrm{HCl}, \mathrm{H}_{2} \mathrm{O}, \mathrm{CO}, \mathrm{CO}_{2}, \mathrm{CH}_{4}, \mathrm{O}_{2}, \mathrm{~N}_{2}$, and He. We subject MOF-5 powder samples to these gas mixtures for pressure cycle testing at $77 \mathrm{~K}$, and to ambient temperature, static exposure testing for 1 week. The hydrogen storage capacity is tested at regular intervals during the pressure cycling, and is tested at the start and end of each static exposure test. Powder XRD and FTIR spectra of the MOF-5 are collected on the post-test samples to check for changes in the crystal structure, and confirm the absence of new phases. The results indicate that common hydrogen fuel impurities at low levels do not lead to significant degradation of MOF-5 powder in either the pressure cycling or ambient static exposure tests.

\section{Experiment details}

\subsection{Materials preparation}

MOF-5 powders were synthesized by BASF at room temperature using a procedure described by Yaghi and coworkers starting from 1,4-benzene dicarboxylic acid $\left(\mathrm{H}_{2} \mathrm{BDC}, \mathrm{C}_{8} \mathrm{H}_{6} \mathrm{O}_{4}\right.$, Merck), zinc acetate dihydrate $\left(\mathrm{Zn}\left(\mathrm{CH}_{3} \mathrm{COO}\right)_{2} \cdot 2 \mathrm{H}_{2} \mathrm{O}\right.$, Merck), and $\mathrm{N}$-dimethylformamide (DMF, BASF $\mathrm{AG})[31]$. In a glass reactor equipped with a Teflon-lined stirrer, $130 \mathrm{~g}$ of $\mathrm{Zn}\left(\mathrm{CH}_{3}-\mathrm{COO}\right)_{2} \cdot 2 \mathrm{H}_{2} \mathrm{O}$ was dissolved in $1200 \mathrm{~mL}$ DMF. Within $2 \mathrm{~h}$, a solution of $37.5 \mathrm{~g}$ of $\mathrm{H}_{2} \mathrm{BDC}$ in $950 \mathrm{~mL}$ DMF was added under vigorous stirring. The precipitate was filtered off, washed three times with $1 \mathrm{~L}$ of dry acetone and dried under a stream of flowing nitrogen. Given the low vapor pressure of DMF (approximately 4 torr at $25^{\circ} \mathrm{C}$ ), solvent exchange to an acetone (vapor pressure of approximately 270 torr at $25^{\circ} \mathrm{C}$ ) has been shown to be an effective method for solvent removal.

Prior to testing and characterization, MOF-5 was heated and evacuated at $130^{\circ} \mathrm{C}$ overnight, yielding the desolvated ('activated') form of the material. Following the initial removal of 
solvent, all handling and storage of MOF-5 was restricted to an active recirculation glovebox with a high-purity Argon atmosphere.

\subsection{Impurity test gases}

The SAE J2719 requirements for fueling stations are summarized in table 1 (Threshold Limit). This standard is based on potential constituents from hydrogen production. It is improbable that a single production method would result in a mixture with all twelve of the contaminants listed in the standard. In addition, preparation of a single gas mixture containing all J2719 contaminants is impractical due to the interactions of the impurities with each other. For example, hydrogen chloride and hydrogen sulfide will react together and with water. Therefore, testing was performed using five separate hydrogen impurity gas mixtures. These were prepared according to the J2719 levels and were based on compositions which could be formulated and qualified by the specialty gas supplier (Airgas). The separate mixtures allowed for independent analysis of the effect of each impurity. Formulation and qualification of the impurity test gas mixtures at the trace levels in SAE J2719 also proved a challenge, necessitating the use of higher impurity levels. Consequently, the following 5 gas mixtures were examined (in all cases the balance of gas is $\left.\mathrm{H}_{2}\right)$ :

Mixture 1: Ammonia $\left(\mathrm{NH}_{3}\right)$, at 5 to $10 \mathrm{ppm}$

Mixture 2: Hydrogen sulfide $\left(\mathrm{H}_{2} \mathrm{~S}\right)$, at $1 \mathrm{ppm}$

Mixture 3: Hydrogen chloride $(\mathrm{HCl})$, at 5 to $10 \mathrm{ppm}$

Mixture 4: Water $\left(\mathrm{H}_{2} \mathrm{O}\right)$, at 5 to $10 \mathrm{ppm}$.

Mixture 5: Carbon monoxide (CO), at $2 \mathrm{ppm}$; Carbon dioxide $\left(\mathrm{CO}_{2}\right)$ at $5 \mathrm{ppm}$; Methane $\left(\mathrm{CH}_{4}\right)$ at 2 ppm; Oxygen $\left(\mathrm{O}_{2}\right)$ at 5 ppm; Nitrogen $\left(\mathrm{N}_{2}\right)$ at 100 ppm; Helium $(\mathrm{He})$ at $500 \mathrm{ppm}$. The 6 impurities present in this mixture are presumed to not react with each other.

The impurity test gas levels are shown in Table 1 . Since the majority of these levels exceed the impurity limits defined in $\mathrm{J} 2719$, the test gas mixtures provide a test of MOF-5 robustness that is more stringent than the $\mathrm{J} 2719$ specification.

\subsection{Cycle test procedures}

Pressure cycling protocols utilized in this study borrow from methods previously used to study conventional hydride materials [32]. Earlier work has established numerous cycle test methods. These include intrinsic cycling, where the same hydrogen gas is re-used each cycle, and extrinsic cycling, where a fresh aliquot of hydrogen is introduced in each cycle. Many of the existing cycle test methods were developed to study aging mechanisms unique to metal hydrides, including alloy disproportionation and particle breakup. The present study employs only extrinsic pressure cycling, as this method best simulates the MOF-5 degradation mechanisms associated with refueling of on-board hydrogen storage systems.

Pressure cycle testing at $77 \mathrm{~K}$ was performed using an automated manometric sorption instrument, following a programmed routine. Each cycle starts with a 5 min adsorption period, where a $168 \mathrm{ml}$ reservoir is filled with a fresh dose of 100-105 bar impurity gas mixture, which 
is then expanded into an evacuated sample cell containing MOF-5. The empty sample cell has a free space of approximately $15 \mathrm{ml}$, with the MOF-5 sample displacing a volume of approximately $200 \mu \mathrm{l}$. At the end of the 5 min adsorption period, the sample cell is closed off. The equilibrium pressure at this point is in the $75-85$ bar range, which corresponds to $5.5-5.8$ wt.\% excess hydrogen at $77 \mathrm{~K}$. This is followed by a 5 min desorption period in which the hydrogen in the sample cell is expanded into an evacuated $1174 \mathrm{ml}$ reservoir. Following the desorption period, the hydrogen pressure in the entire instrument is slowly ramped down to 1 bar, and then opened to continuous vacuum for $5 \mathrm{~min}$. During the active vacuum period nearly all of the adsorbed hydrogen should be removed. This cycle pattern is repeated 300 times in total for each test gas. Cycling is interrupted approximately every 60 cycles to measure the hydrogen adsorption capacity of the sample.

Pressure cycle tests were conducted for all five test gas mixtures listed in Table 1. Throughout each period of continuous cycling the sample cell is immersed in a liquid nitrogen $\left(\mathrm{LN}_{2}\right)$ bath, and is allowed to warm up only for the capacity test. To prevent MOF-5 powder from being blown out of the sample cell during the repeated desorption steps, which involve a large pressure differential, a 2 micron sintered metal filter gasket was placed immediately above the sample cell. The filter has a 0.125 inch hole drilled in it to allow an internal PRT sensor to extend into the sample cell. Fresh samples of MOF-5 powder were used for each gas mixture test and were retained for the entire 300 cycles. 
initially immersed in liquid nitrogen. The sample cell was then valved off and allowed to warm to room temperature. It was left under pressure (above 100 Bar) at room temperature for 1 week. Storage tests were performed for the $\mathrm{H}_{2} \mathrm{O}$ and $\mathrm{H}_{2} \mathrm{~S}$ test gas mixtures.

\subsection{Capacity test procedure}

Capacity tests consist of measuring a single hydrogen adsorption isotherm at $77 \mathrm{~K}$, up to a maximum pressure of approximately 90 bar. Hydrogen adsorption measurements were performed using a manometric sorption instrument (PCT-Pro 2000, Setaram) with an oil-free scroll vacuum pump (Anest Iwata model ISP90). Free space measurements were performed using helium at room temperature for each sample. The sample cell was immersed in a liquid nitrogen bath, with the $\mathrm{LN}_{2}$ level filled to a specific height. Excess adsorption amounts were calculated by the standard method [33]. Further details on the isotherm measurement methods can be found in Ref [29].

Initial capacity tests were collected prior to starting the pressure cycling and storage procedures. Before being loaded in the sample cell, MOF-5 powder samples were degassed to remove any weakly-bound water. Samples were evacuated at room temperature for at least 6 hours, then evacuated and heated to $130{ }^{\circ} \mathrm{C}$ overnight.

Final capacity tests were collected at the end of both pressure cycling and storage. For pressure cycling, capacity tests were measured approximately every 60 cycles until the end of the 300 cycle tests. With the exception of the initial capacity tests, the MOF-5 samples were degassed only by pulling vacuum on the sample at room temperature (no heating) before capacity tests. (For the initial capacity tests the freshly loaded MOF-5 sample was degassed at $130^{\circ} \mathrm{C}$, as described in the previous paragraph.)

Settings, dose increments, and step times were identical for all capacity test measurements. Due to the difficulty in switching test gas bottles in the middle of storage and pressure cycle testing, capacity tests were performed using the same impurity gas mixtures used in the corresponding cycle and storage procedures. Throughout this document, the unit of weight percent (wt. \%) refers to the mass of adsorbed hydrogen per $100 \mathrm{~g}$ of adsorbent. (The mass of adsorbed hydrogen is not included in the denominator.)

\subsection{XRD and FTIR characterization}

Powder X-ray diffraction (XRD, Rigaku Miniflex II diffractometer using $\mathrm{Cu} \mathrm{K}-\alpha$ radiation ( $\lambda$ $=1.5418 \AA$ )) was used to assess changes in crystallinity resulting from exposure to testing gas containing impurities. Samples were transferred to the diffractometer in sealed vials. Each vial was opened, and the MOF-5 powder was chopped and pressed onto an off-axis silicon crystal sample substrate. The time between opening the vial and the beginning of the scan was less than 30 seconds. Diffraction data was collected over a range of $5-40^{\circ} 2 \theta$ at a scan rate of $5 \% \mathrm{~min}$, resulting in a total experiment time of 7 minutes. The sample was then immediately scanned a second time in order to observe the degree of structural change that had occurred during the initial scan. No change was observed in any of the samples. 
Fourier transform infrared spectrometry (FTIR) was used to assess changes in bonding resulting from exposure to impurities. Measurements were made using a Thermo Scientific Nicolet FTIR spectrometer. A scan range of 4000 to $600 \mathrm{~cm}^{-1}$ was used, with a scan time of 5 minutes. Powder samples were loaded and scanned in air. As demonstrated previously [24], the MOF-5 powders used in this study do not show structure change when exposed to air for less than 5 minutes. We expect that any changes to FTIR peaks primarily reflect effects from exposure to impurities in the testing gas.

\section{Results and Discussion}

\subsection{Impurity cycle test}

\subsubsection{Ammonia}

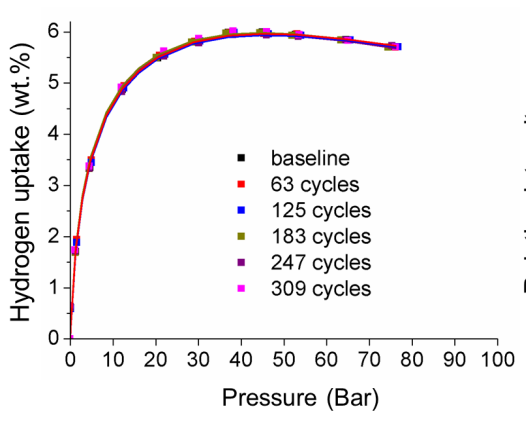

(a)

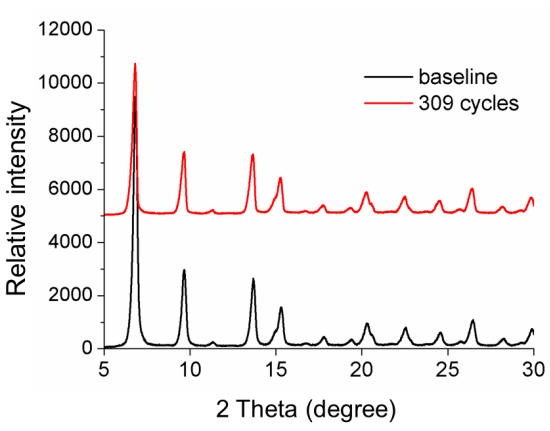

(b)

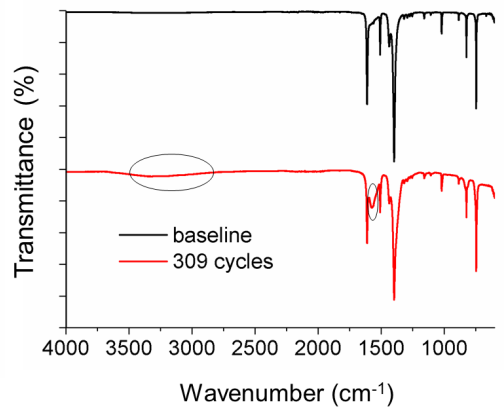

(c)

Figure 2. (a) Adsorption isotherms for MOF-5 powders with test gas mixture 1, which contains $\mathrm{NH}_{3}$ as the impurity. Capacity measurement are taken every 60 cycles. (b) XRD spectra for MOF-5 powders before and after the cycle test. (c) FTIR spectra for MOF-5 powders before and after the cycle test.

The ammonia impurity mixture (Mixture 1) contains a concentration of $7 \mathrm{ppm}$, which is roughly 70 times larger than the $0.1 \mathrm{ppm}$ threshold in J2719. Hydrogen capacity tests measured on the MOF-5 sample during pressure cycling with the ammonia test gas are summarized in figure 2(a). The adsorption isotherms after 63, 125, 183, 247, 309 cycles overlap exactly (within measurement error), indicating no hydrogen uptake capacity loss after pressure cycle testing.

The powder XRD spectra shown in figure 2(b), measured before and after cycle testing, fails to detect any significant change to the long-range crystal structure due to the cycling. Nevertheless, two small changes are present in the FTIR spectra shown in figure 2(c). These changes correspond to the emergence of two new peaks: The broad, weak peak around $3300 \mathrm{~cm}^{-1}$ can be attributed to $\mathrm{O}-\mathrm{H}$ bond stretching in carboxylic acid, while the peak near $1600 \mathrm{~cm}^{-1}$ arises from the $\mathrm{C}=\mathrm{O}$ bond in carboxylic acid. A possible reaction signaled by the emergence of these peaks is breaking of the $\mathrm{Zn}-\mathrm{O}$ bond between the metal cluster and organic linker, with a proton from $\mathrm{NH}_{3}$ combining with $\mathrm{COO}^{-}$to form carboxylic acid. It appears as though this reaction only effects a 
small fraction of Zn-O bonds, given that neither the powder XRD spectrum nor the hydrogen storage capacity are altered. This maybe because the amount of impurities accumulated during the cycle test is small, and can only affect a similarly small fraction of the Zn-O bonds in the sample.

\subsubsection{Hydrogen Chloride (Halogenates)}

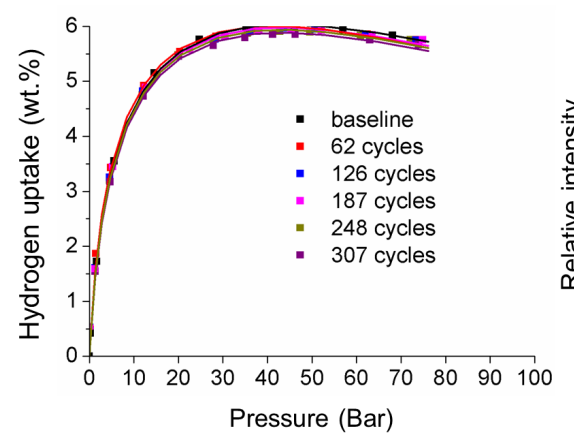

(a)

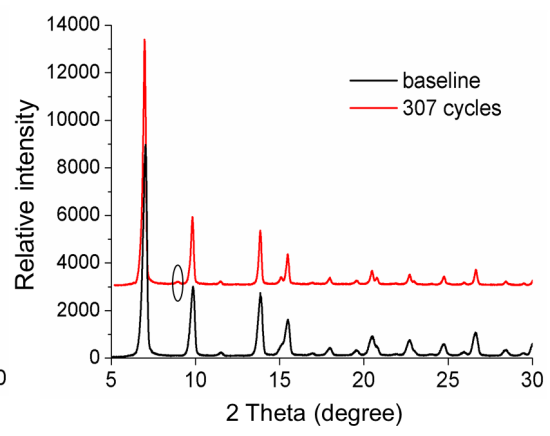

(b)

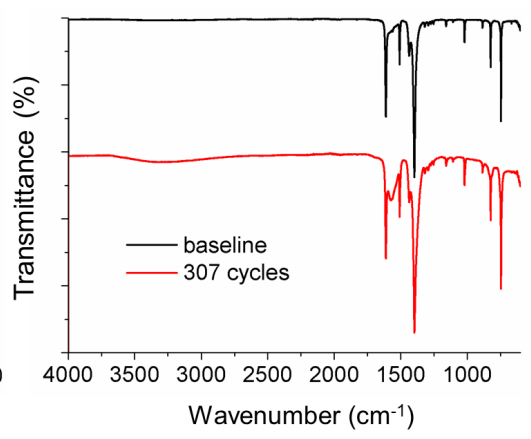

(c)

Figure 3. (a) Adsorption isotherms for MOF-5 powders with test gas mixture 3, which contains $\mathrm{HCl}$ as the impurity. Capacity measurement are taken every 60 cycles. (b) XRD spectra for MOF-5 powders before and after the cycle test. (c) FTIR spectra for MOF-5 powders before and after the cycle test.

The characterization results of MOF-5 powder before and after pressure cycling with hydrogen chloride are shown in figure 3. As noted in Sec. 2, the $9 \mathrm{ppm}$ impurity concentration of hydrogen chloride in this hydrogen test gas (Mixture 3) is 180 times larger than the $0.05 \mathrm{ppm}$ threshold limit in J2719. Despite these relatively high levels, hydrogen adsorption isotherms in figure 3(a) indicate only a small apparent decrease in hydrogen uptake after 307 cycles. The maximum excess $\mathrm{H}_{2}$ adsorption amount at $77 \mathrm{~K}$ decreases $2.5 \%$ from 6.0 wt.\% to $5.85 \mathrm{wt} \%$ (excess) after 307 cycles.

Further characterization confirms that this capacity loss can be traced to MOF-5 structure decomposition. The powder XRD pattern of the post-cycling sample has a new peak emerging at $2 \theta=9^{\circ}$, as shown in figure 3(b). This peak also appears in the powder XRD spectrum for MOF-5 following water-induced structure degradation [20]. The emergence of the $9^{\circ} \mathrm{C}$ peak indicates degradation and/or amorphization of the MOF-5 crystal structure. In addition to this change in the XRD patter, the FTIR spectrum in figure 3(c) shows the same impurity-induced peaks at $3300 \mathrm{~cm}^{-1}$ and $1600 \mathrm{~cm}^{-1}$ that are present for the ammonia-containing test gas in figure 2 (c). This suggests a common reaction pathway leading to the structure decomposition of MOF-5 following exposure to ammonia, hydrogen chloride, and water impurities.

\subsubsection{Water, Hydrogen Sulfide (Sulfur), and Inerts Mixture}


Results for the remaining three mixtures (2, 4 and 5 in Table 1) are summarized in the Supporting Information, in figures S1, S2 and S3, respectively. Mixture 2 contains hydrogen sulfide, while mixture 4 contains water. Mixture 5, which includes a set of mostly inert impurity species is denoted "inerts" for identification. For all three of these mixtures there was no measurable change in the hydrogen adsorption capacity tests measured during the course of pressure cycling. Likewise, for all three impurity mixtures the MOF-5 crystal structure and bonding network appears unchanged following 300 pressure cycles. The powder XRD patterns (figures S1b, S2b, S3b) and FTIR spectra (figures S1c, S2c, S3c) collected on the pre-cycling and post-cycling MOF-5 samples do not show any significant changes. Since the impurity concentrations in Mixtures 2, 4 and 5 significantly exceed the threshold levels specified in J2719, we conclude that these contaminants will not impact the adsorption capacity of MOF-5 powders within 300 cycles.

\subsubsection{Summary of pressure cycling tests}

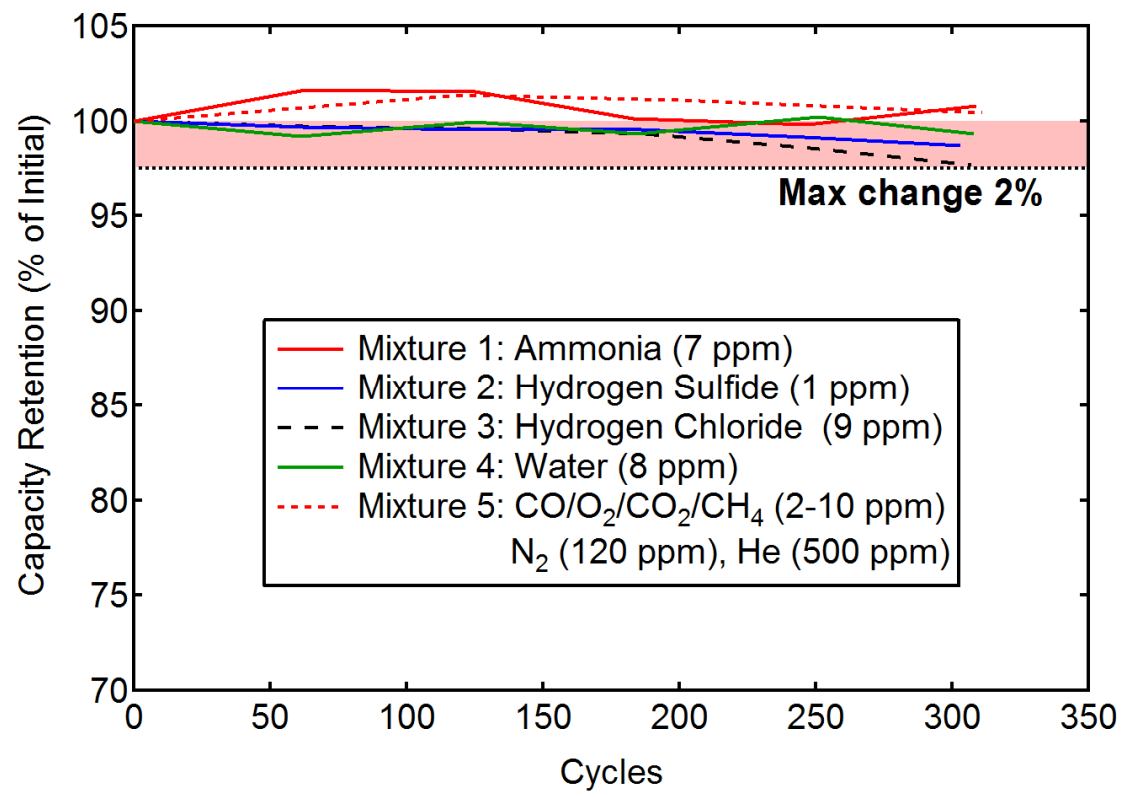

Figure 4. Summary of maximum hydrogen excess adsorption in MOF-5 (expressed as a percentage of the maximum adsorption before the start of testing) measured at $77 \mathrm{~K}$ and 80 bar as a function of cycle number with impurity gas mixtures.

Figure 4 provides a summary of the hydrogen capacity measured during pressure cycling tests for all five impurity gas mixtures. We reiterate that each hydrogen adsorption capacity test consisted of measuring an excess adsorption isotherm at $77 \mathrm{~K}$, using the same impurity gas employed in the ongoing cycling sequence. The capacity is therefore defined in terms of the maximum excess adsorption at $77 \mathrm{~K}$. The hydrogen capacities plotted on the y-axis in Figure 4 are expressed as a percentage of the initial capacity measured at the beginning of the cycle 
testing (i.e., capacity at cycle 0). For all five of the impurity gases tested, the capacity retention was above $97 \%$ after 300 cycles.

\subsection{Static exposure tests}

\subsubsection{Water}

A one-week static exposure test was performed at room temperature using the water impurity mixture (mixture 4), and was carried out according to the procedure described in section 2. Figure 5 summarizes the pre- and post-storage characterization results for this MOF-5 sample. The hydrogen uptake curves shown in figure 5a indicate that there was no adsorption capacity change following the one-week exposure. The XRD and FTIR profiles also indicate a stable bonding and crystal structure of MOF-5 samples following the test. When combined with the earlier pressure cycling test results, the static exposure test results confirm that $\mathrm{H}_{2} \mathrm{O}$ concentrations up to $8 \mathrm{ppm}$ in the hydrogen fuel do not affect hydrogen storage performance of MOF-5 within 300 cycles and 1 week exposure. According to $\mathrm{J} 2719$ standard, the content of $\mathrm{H}_{2} \mathrm{O}$ should be less than 5 ppm, which presents an even safer threshold.

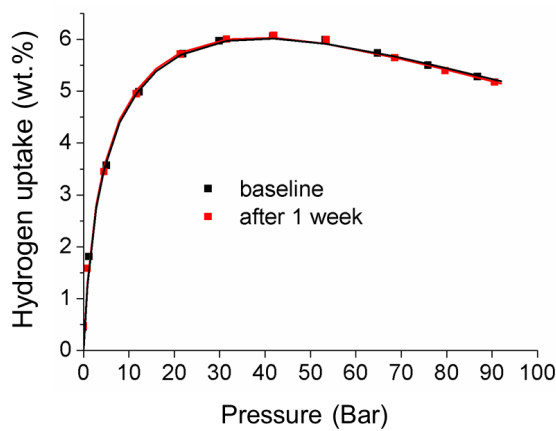

(a)

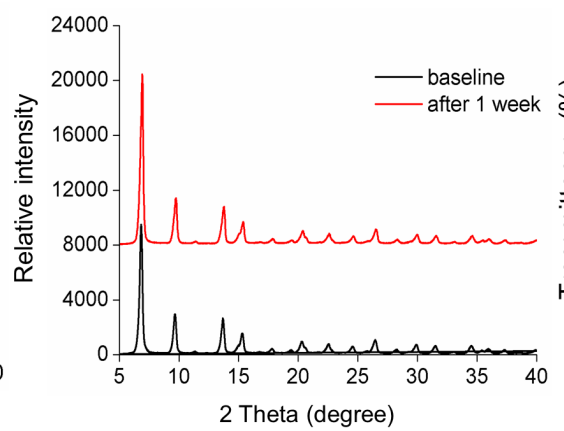

(b)

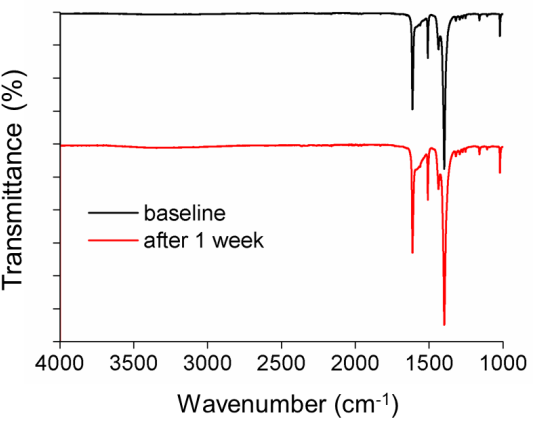

(c)

Figure 5. (a) Adsorption isotherms for MOF-5 powders with test gas mixture 4, which contains $\mathrm{H}_{2} \mathrm{O}$ as the impurity. Capacity measurement are taken before and after 1 week static exposure. (b) XRD spectra for MOF-5 powders before and after static exposure. (c) FTIR spectra for MOF-5 powders before and after static exposure.

\subsubsection{Hydrogen Sulfide (Sulfur)}




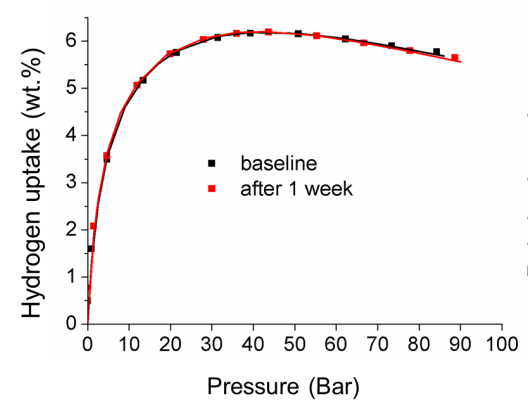

(a)

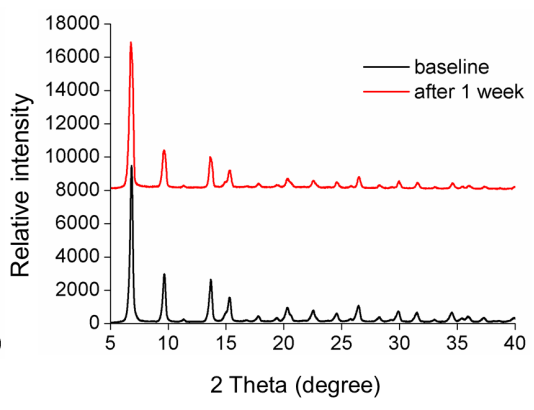

(b)

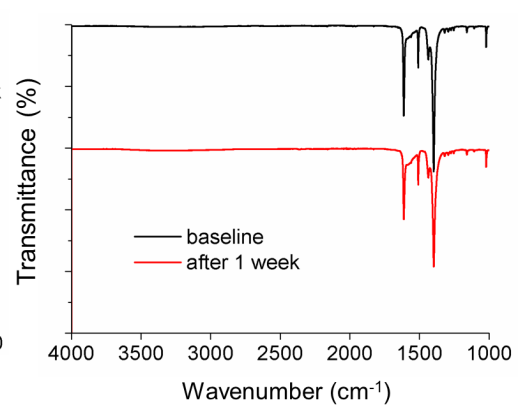

(c)

Figure 6. (a) Adsorption isotherms for MOF-5 powders with test gas mixture 2, which includes $\mathrm{H}_{2} \mathrm{~S}$ as the impurity. Capacity measurement are taken before and after 1 week static exposure. (b) XRD spectra for MOF-5 powders before and after static exposure. (c) FTIR spectra for MOF-5 powders before and after static exposure.

MOF-5 was subjected to a one-week static exposure test at room temperature using the hydrogen sulfide impurity mixture (mixture 2). Figure 6 summarizes the characterization results of the MOF-5 powder before and after the test. The hydrogen uptake isotherms in figure 6(a) indicate no decrease in adsorption capacity. The unchanged peaks in XRD and FTIR in figures 6 (b) \& (c) also show that the bonding network and crystal structure of MOF-5 samples are not altered during the exposure test. Combining the results of the cycle test and static exposure test we conclude that $0.9 \mathrm{ppm} \mathrm{H}_{2} \mathrm{~S}$ in hydrogen does not significantly affect the hydrogen storage performance of MOF-5 within 300 cycles and 1 week of static exposure. According to the $\mathrm{J} 2719$ standard, the concentration of $\mathrm{H}_{2} \mathrm{~S}$ in the hydrogen fuel stream should be less than $0.004 \mathrm{ppm}$. This level is more than 200 times lower than in the test mixture, signaling that a gas stream that meets the specification will be even less likely to result in degradation of MOF-5.

\section{Conclusion}

Hydrogen fuel conforming to purity standards outlined in SAE J2719 can contain contaminants. We studied the effect of these impurities on the hydrogen storage capacity, bonding, and crystal structure of the prototype metal-organic framework, MOF-5 using cyclic and static exposure tests. Four impure hydrogen gas mixtures were prepared by introducing ammonia, hydrogen sulfide, hydrogen chloride, and water, respectively. Six additional (mostly inert) impurities were combined into a fifth hydrogen mixture. Pressure cycle testing at $77 \mathrm{~K}$ with these fuel streams revealed that only the hydrogen chloride mixture yielded a measurable decrease in hydrogen storage capacity (from $6 \mathrm{wt} . \%$ to $5.8 \mathrm{wt} . \%$.). No measurable decrease was noted for the other mixtures during cycling. Cycling with hydrogen chloride-containing mixture also induced changes to the MOF-5 crystal structure and its local bonding network, as 
determined by XRD and FTIR, respectively. Changes to the bonding structure were also observed for samples cycled with trace ammonia, but corresponding changes to the long-range crystal structure or hydrogen storage capacity could not be detected. Static exposure of MOF-5 to water and hydrogen sulfide impurities had no measurable effect on the sample.

We emphasize that the impurity levels used in this study exceed the J2719 threshold by at least of a factor of 10, and in some cases exceed it by a factor of 200. The robustness observed at these higher concentrations demonstrate that hydrogen from a fueling station compliant with J2719 should not impact the performance of a MOF-5-based hydrogen storage system for up to 300 cycles and 1 week's static exposure.

\section{ASSOCIATED CONTENT}

Supporting Information. Hydrogen adsorption isotherms, X-ray diffraction and Fourier transform infrared spectroscopy spectra for cycle tests involving impure hydrogen fuel streams containing $\mathrm{H}_{2} \mathrm{~S}, \mathrm{H}_{2} \mathrm{O}$, and a mixture of $\mathrm{CO}, \mathrm{CO}_{2}, \mathrm{CH}_{4}, \mathrm{O}_{2}, \mathrm{~N}_{2}$, and $\mathrm{He}$.

\section{AUTHOR INFORMATION}

\section{Corresponding Author}

*djsiege@umich.edu; +1 734-764-4808 (tel); +1 734-764-4256 (fax)

\section{ACKNOWLEDGMENTS}

Funding for this study was provided by the U.S. Department of Energy, Office of Energy Efficiency and Renewable Energy, award number DE-FC36-GO19002.

\section{References}

[1] U. Mueller, M. Schubert, F. Teich, H. Puetter, K. Schierle-Arndt, J. Pastre, Metal-organic frameworks-prospective industrial applications, Journal of Materials Chemistry, 16(7) (2006) 626-636.

[2] K. Schlichte, T. Kratzke, S. Kaskel, Improved synthesis, thermal stability and catalytic properties of the metal-organic framework compound Cu3(BTC)2, Microporous and Mesoporous Materials, 73(1-2) (2004) 81-88.

[3] O.M. Yaghi, M. O'Keeffe, N.W. Ockwig, H.K. Chae, M. Eddaoudi, J. Kim, Reticular synthesis and the design of new materials, Nature, 423(6941) (2003) 705-714.

[4] L. Bellarosa, S. Calero, N. Lopez, Early stages in the degradation of metal-organic frameworks in liquid water from first-principles molecular dynamics, Physical Chemistry Chemical Physics, 14(20) (2012) 7240-7245.

[5] L. Bellarosa, J.M. Castillo, T. Vlugt, S. Calero, N. López, On the Mechanism Behind the Instability of Isoreticular Metal-Organic Frameworks (IRMOFs) in Humid Environments, Chemistry - A European Journal, 18(39) (2012) 12260-12266.

[6] M. De Toni, R. Jonchiere, P. Pullumbi, F.-X. Coudert, A.H. Fuchs, How Can a Hydrophobic MOF be Water-Unstable? Insight into the Hydration Mechanism of IRMOFs, ChemPhysChem, 13(15) (2012) 3497-3503.

[7] J.A. Greathouse, M.D. Allendorf, The Interaction of Water with MOF-5 Simulated by Molecular Dynamics, Journal of the American Chemical Society, 128(33) (2006) 10678-10679. 
[8] S. Han, Y. Huang, T. Watanabe, S. Nair, K.S. Walton, D.S. Sholl, J. Carson Meredith, MOF stability and gas adsorption as a function of exposure to water, humid air, SO2, and NO2, Microporous and Mesoporous Materials, 173(0) (2013) 86-91.

[9] H. Jasuja, N.C. Burtch, Y.G. Huang, Y. Cai, K.S. Walton, Kinetic Water Stability of an Isostructural Family of Zinc-Based Pillared Metal-Organic Frameworks, Langmuir, 29(2) (2013) 633-642.

[10] S.S. Kaye, A. Dailly, O.M. Yaghi, J.R. Long, Impact of Preparation and Handling on the Hydrogen Storage Properties of $\mathrm{Zn} 4 \mathrm{O}(1,4-$ benzenedicarboxylate)3 (MOF-5), Journal of the American Chemical Society, 129(46) (2007) 14176-14177.

[11] J.J. Low, A.I. Benin, P. Jakubczak, J.F. Abrahamian, S.A. Faheem, R.R. Willis, Virtual High Throughput Screening Confirmed Experimentally: Porous Coordination Polymer Hydration, Journal of the American Chemical Society, 131(43) (2009) 15834-15842.

[12] C. Petit, B. Mendoza, T.J. Bandosz, Hydrogen Sulfide Adsorption on MOFs and MOF/Graphite Oxide Composites, ChemPhysChem, 11(17) (2010) 3678-3684.

[13] J.J. Gutierrez-Sevillano, A. Martin-Calvo, D. Dubbeldam, S. Calero, S. Hamad, Adsorption of hydrogen sulphide on Metal-Organic Frameworks, RSC Advances, 3(34) (2013) 14737-14749.

[14] L. Hamon, C. Serre, T. Devic, T. Loiseau, F. Millange, G. Férey, G.D. Weireld, Comparative Study of Hydrogen Sulfide Adsorption in the MIL-53(Al, Cr, Fe), MIL-47(V), MIL-100(Cr), and MIL-101(Cr) Metal-Organic Frameworks at Room Temperature, Journal of the American Chemical Society, 131(25) (2009) 8775-8777.

[15] D. Saha, S. Deng, Ammonia adsorption and its effects on framework stability of MOF-5 and MOF-177, Journal of Colloid and Interface Science, 348(2) (2010) 615-620.

[16] C. Petit, T.J. Bandosz, Enhanced Adsorption of Ammonia on Metal-Organic Framework/Graphite Oxide Composites: Analysis of Surface Interactions, Advanced Functional Materials, 20(1) (2010) 111-118.

[17] L. Huang, T. Bandosz, K.L. Joshi, A.C.T. van Duin, K.E. Gubbins, Reactive adsorption of ammonia and ammonia/water on CuBTC metal-organic framework: A ReaxFF molecular dynamics simulation, The Journal of Chemical Physics, 138(3) (2013) 034102.

[18] L. Ding, A.Ö. Yazaydin, How Well Do Metal-Organic Frameworks Tolerate Flue Gas Impurities?, The Journal of Physical Chemistry C, 116(43) (2012) 22987-22991.

[19] L. Valenzano, B. Civalleri, S. Chavan, G.T. Palomino, C.O. Areán, S. Bordiga, Computational and Experimental Studies on the Adsorption of CO, N2, and CO2 on Mg-MOF-74, The Journal of Physical Chemistry C, 114(25) (2010) 11185-11191.

[20] Y. Ming, J. Purewal, J. Yang, C. Xu, R. Soltis, J. Warner, M. Veenstra, M. Gaab, U. Müller, D.J. Siegel, Kinetic Stability of MOF-5 in Humid Environments: Impact of Powder Densification, Humidity Level, and Exposure Time, Langmuir, 31 (2015) 4988-4995.

[21] S. International, Hydrogen Fuel Quality for Fuel Cell Vehicles, (2011).

[22] http://www.nrel.gov/hydrogen/images/cdp_infr_25.jpg.

[23] R. Chahine, T.K. Bose, Characterization and optimization of adsorbents for hydrogen storage, Hydrogen Energy Progress Xi, Vols 1-3, (1996) 1259-1263.

[24] Y. Ming, J. Purewal, D.a. Liu, A. Sudik, C. Xu, J. Yang, M. Veenstra, K. Rhodes, R. Soltis, J. Warner, M. Gaab, U. Müller, D.J. Siegel, Thermophysical properties of MOF-5 powders, Microporous and Mesoporous Materials, 185(0) (2014) 235-244. 
[25] J.J. Purewal, D. Liu, J. Yang, A. Sudik, D.J. Siegel, S. Maurer, U. Müller, Increased volumetric hydrogen uptake of MOF-5 by powder densification, International Journal of Hydrogen Energy, 37(3) (2012) 2723-2727.

[26] C. Xu, J. Yang, M. Veenstra, A. Sudik, J.J. Purewal, Y. Ming, B.J. Hardy, J. Warner, S. Maurer, U. Müeller, D.J. Siegel, Hydrogen permeation and diffusion in densified MOF-5 pellets, International Journal of Hydrogen Energy, 38(8) (2013) 3268-3274.

[27] Y. Ming, H. Chi, R. Blaser, C. Xu, J. Yang, M. Veenstra, M. Gaab, U. Müller, C. Uher, D.J. Siegel, Anisotropic thermal transport in MOF-5 composites, International Journal of Heat and Mass Transfer, 82(0) (2015) 250-258.

[28] D. Liu, J.J. Purewal, J. Yang, A. Sudik, S. Maurer, U. Mueller, J. Ni, D.J. Siegel, MOF-5 composites exhibiting improved thermal conductivity, International Journal of Hydrogen Energy, 37(7) (2012) 6109-6117.

[29] J. Purewal, D. Liu, A. Sudik, M. Veenstra, J. Yang, S. Maurer, U. Müller, D.J. Siegel, Improved Hydrogen Storage and Thermal Conductivity in High-Density MOF-5 Composites, The Journal of Physical Chemistry C, 116(38) (2012) 20199-20212.

[30] J. Goldsmith, A.G. Wong-Foy, M.J. Cafarella, D.J. Siegel, Theoretical Limits of Hydrogen Storage in Metal-Organic Frameworks: Opportunities and Trade-Offs, Chemistry of Materials, 25(16) (2013) 3373-3382.

[31] D.J. Tranchemontagne, J.R. Hunt, O.M. Yaghi, Room temperature synthesis of metal-organic frameworks: MOF-5, MOF-74, MOF-177, MOF-199, and IRMOF-0, Tetrahedron, 64(36) (2008) 8553-8557.

[32] D. Chandra, Chapter 12, Solid State Hydrogen Storage: Materials and Chemistry, CRC Press, LLC: Boca Raton, U.S.A, (2008).

[33] M.-A. Richard, D. Cossement, P.-A. Chandonia, R. Chahine, D. Mori, K. Hirose, Preliminary evaluation of the performance of an adsorption-based hydrogen storage system, AIChE Journal, 55(11) (2009) 2985-2996. 